\title{
Metabolic Bone Disease in Chronic Kidney Disease Patients (CKD-MBD) and its Associated Factors at Muhimbili National Hospital in Dar es salaam, Tanzania: A Cross-Sectional Study
}

Felix F. Mungulluh ${ }^{1 \S}$, Paschal Ruggajo ${ }^{1,2}$, Francis F. Furia ${ }^{1}{ }^{2}$

${ }^{1}$ School of Medicine, Muhimbili University of Health and Allied Sciences, Dar es SalaamTanzania

${ }^{2}$ Renal Unit, Muhimbili National Hospital, Dar es Salaam-Tanzania

Email addresses:

PR: prugajo@yahoo.com

FFF: fredrick.francis78@gmail.com

FFM: felix.faustin40@gmail.com

${ }^{\S}$ Corresponding Author

Corresponding author's address:

Felix Faustin Mungulluh, MD

felix.faustin40@gmail.com

$+255714025620$ 


\section{ABSTRACT}

Background: Metabolic bone disease in chronic kidney disease patients is associated with high morbidity and mortality, and it has been reported to start early in the course of the disease and worsen as the kidney damage progresses. However, the prevalence and factors associated with metabolic bone disease in chronic kidney disease patients in our setting has not been established, so we aimed to determine the prevalence and factors associated with metabolic bone disease among patients with chronic kidney disease at a tertiary Muhimbili National Hospital in Dar es salaam, Tanzania so as to help physicians recognize the patients at risk, diagnose the problem and prevent complications sooner.

Methods: This was a hospital based cross-sectional study involving adult patients with chronic kidney disease attending renal unit Muhimbili National Hospital, a tertiary referral center in Dar es Salaam, Tanzania. Systematic sampling technique was employed to get study participants after being given informed consent. In this study, CKD-MBD was defined basing on the abnormality of serum calcium, phosphate or parathyroid hormone level. Data analysis was done using the SPSS version 23.0 software.

Results: A total of 300 participants with chronic kidney disease stage 3 and above were included in this study. Majority were male, 198 (66.0\%), with a mean age of 53 years. The prevalence of metabolic bone disease was found to be $75.0 \%$. The most common form of metabolic bone disease was hyperparathyroidism 196 (87.1\%), followed by hypocalcemia 174 (77.3\%) and hyperphosphatemia 82 (36.4\%), which was the least common.

Factors which were found to be significantly associated with CKD-MBD were the use of calcium supplements, use of phosphate binders, being on dialysis, a calcium rich diet and a low phosphate diet.

Conclusion: Metabolic bone diseases are common in patients with CKD at Muhimbili National Hospital. Patients with CKD should undergo serial assessment of phosphate, calcium and parathyroid hormone level, considered together, so as to diagnose metabolic bone disease early and treat those patients who will be found to have persistently or prolonged abnormalities in these bone mineral biomarkers.

Keywords: Metabolic bone disease, chronic kidney disease, hyperparathyroidism, hypocalcemia, hyperphosphatemia 


\section{Background}

Chronic kidney disease (CKD) is associated with metabolic bone disease which starts early in the course of the disease and worsens as the kidney damage progress [1]. Globally, chronic kidney disease-metabolic bone disorder (CKD-MBD) among patients with end stage renal disease ranges from $33 \%$ to $67 \%$, and its severity tends to increase with the progression of the kidney damage, and is associated with high mortality rates [2].

As the kidney functions decline, there is a decrease in phosphate elimination, leading to hyperphosphatemia and a hypocalcemia. Both hypocalcemia and hyperphosphatemia stimulate the secretion of parathyroid hormone (PTH). In an attempt to normalize ionized calcium and serum phosphate levels, PTH increases reabsorption of calcium in the distal tubules and decreases phosphate reabsorption in the proximal tubules of the kidney and also increases calcium mobilization from bones in early stages of CKD (Tradeoff hypothesis) [3]. Eventually estimated glomerular filtration rate (eGFR) gets so low that even high levels of PTH cannot increase phosphate excretion and normalize calcium level leading to hyperphosphatemia and hypocalcemia, respectively, causing further elevation of PTH level.

Several factors have been associated with CKD-MBD, including; age, sex, type and duration of dialysis, use of phosphate binders, use of calcimimetics and vitamin D analog, parathyroidectomy, history of diabetes and hypertension, duration of kidney failure and differences in dietary habits [4-8]. Countries in sub-Saharan Africa including Tanzania have documented growing burden of CKD but there is limited information on CKD-MBD among these patients. This study was aimed at determining the prevalence of CKD-MBD and its risk factors among CKD patients at Muhimbili National Hospital in Dar es Salaam, Tanzania so as to help physicians recognize the patients at risk, diagnose the problem and prevent complications sooner. In this study, CKD-MBD was defined basing on the abnormality of serum calcium, phosphate or parathyroid hormone level [9].

\section{Methods}

\section{Study design and setting}

This was a hospital based cross sectional study conducted among CKD adult patients attending nephrology outpatient clinic at MNH from May to December 2019. MNH is the largest referral hospital in Tanzania, with bed capacity of 1500, it also serve as teaching hospital of Muhimbili University of Health and Allied Sciences (MUHAS). MNH receives referral patients from both 
public and private hospitals from all over the country. The renal clinic at MNH operates once a week (on Wednesdays) receiving an average of 40 patients per clinic day.

\section{Study Population and Sample size}

All adult CKD patients aged 18 years and above attending renal clinic at MNH were eligible. Sample size for this study was calculated based on prevalence of CKD-MBD which is obtained from the study done by Mugera at Kenyatta National Hospital (2013), where prevalence was found to be $22.4 \%$ [32]. Leslie Kish formula was used to determine the sample size of the study participants where a minimum sample size of 300 participants was obtained.

\section{Sampling method}

Participants were identified using a systematic sampling technique, where the list of patients was obtained from the appointment book, creating a sampling frame. Sampling interval was obtained to be 3 . The first person was randomly selected from the sampling frame between 1 to 3 and then every third patient was selected and screened for the inclusion and exclusion criteria to identify eligible individuals to be enrolled in the study and was included only once.

\section{Data collection methods}

Data was collected using an interviewer administered structured Clinical Research Form (CRF). Principal investigator and research assistants conducted face-to-face interviews and physical examination. Data collected included socio-demographic data, clinical history, duration and type of CKD treatment (conservative, dialysis or concomitant medications), duration of dialysis and type of diet. Results of laboratory tests including serum creatinine, blood urea nitrogen, calcium, phosphate, parathyroid hormone and albumin level were also recorded into the CRF.

\section{Laboratory testing}

About $5 \mathrm{ml}$ of blood was drawn from the cubital vein into sterile plain vacutainer tubes for serum creatinine, blood urea nitrogen, calcium, phosphate, parathyroid hormone and albumin level. The blood samples were immediately processed and if there was a delay, they were then stored at -20 degree Celsius until the time of processing. The measurements of serum creatinine, blood urea nitrogen, calcium, phosphate and albumin were determined using an automatic chemistry analyzer Biotecnica 3500 (BT3500) while serum intact parathyroid hormone levels were determined using electro-chemiluminescence immunoassay Maglumi800at MNH laboratory. The results were analyzed after daily calibration using standard calibration methods and materials and tests assayed against controls. 
Total serum calcium was corrected for serum albumin using the equation:

Corrected serum calcium $=$ measured serum calcium +0.02 (40- Serum Albumin) [10] .

The Glomerular Filtration Rate (GFR) was estimated using the Modification of Diet in Renal

Disease (MDRD) formula as follows [11]:

$\mathrm{eGFR}=186 \times(\text { Serum Creatinine/88.4 })^{-1.154} \mathrm{x}(\text { Age })^{-0.203} \times[1.212$ if Black $] \times[0.742$ if Female $]$.

\section{Data management and analysis}

Data was entered into the Statistical Package for Social Sciences (SPSS version 23.0) for analysis. The prevalence of CKD-MBD was determined by taking the proportion of enrolled patients who have abnormalities of serum markers (calcium, phosphate or parathyroid hormone levels). Categorical variables were summarized as frequencies and percentages, and continuous variables as means and standard deviation. Categorical and continuous variables were compared with the chi-square and t-test, respectively. The association between the categorical independent variables and CKD-MBD was determined using univariate logistic regression. To control for confounding those factors with $\mathrm{p} \leq 0.2$ were subjected to a multivariate logistic regression. Pvalue of $<0.05$ was considered statistically significant.

\section{Ethical considerations}

The study protocol was reviewed and approved by the Institution of Review Board of the Muhimbili University of Health and Allied Sciences (reference number DA.287/298/01A) and permission to collect data was obtained from relevant authorities at MNH. All participants were provided a written informed consent prior to participation into the study. 


\section{Results}

\section{Socio-demographic and clinical characteristics of the study participants}

A total of 300 participants with CKD stage 3-5 were recruited into this study. Majority were male, $198(66 \%)$, with a mean age of $53.7 \pm 12.0$ years. More than half were over 55 years. Atleast half of the patients 151 (50.3\%) had been informed of their renal disease less than 2 years prior to this study. Majority of participants 284 (94.7\%) were in CKD stages (4, 5 and 5D) and $129(43.0 \%)$ were on maintenance hemodialysis. Most participants had hypertension 285 (95\%). Diabetes and hypercholesterolemia were noted in 77 (25.7\%) and 16 (5.3\%) participants respectively (Table 1). 
Table 1: Socio-demographic and clinical characteristics of the study participants, $\mathrm{N}=300$

\begin{tabular}{lll}
\hline Variable & Frequency (N) & Percentage (\%) \\
\hline Age group & 32 & \\
18-35 & 101 & 10.7 \\
36-55 & 167 & 33.7 \\
Above 55 & $53.7 \pm 12.0$ & 55 \\
Mean age & & \\
Gender & 198 & 66.0 \\
Male & 102 & 34.0 \\
Female & & \\
Marital status & 50 & 16.7 \\
Single & 250 & 83.3 \\
Living with a partner & & \\
Education level & 13 & 4.3 \\
Non-formal education & 287 & 95.7 \\
Formal education & & \\
Occupation & 45 & 15.0 \\
Unemployed & 255 & 85.0 \\
Employed & & \\
Duration of CKD (Years) & 151 & 50.3 \\
<2 & 149 & 49.7 \\
Z2 CKD stage & & \\
Early (3A,3B) & 16 & 5.3 \\
Late (4,5,5D) \\
eGFR mean \\
RRT
\end{tabular}




\section{The prevalence of CKD-MBD}

The prevalence of CKD-MBD among all participants was found to be $75 \%$ and majority of patients on maintenance hemodialysis (CKD stage 5D) and CKD stage 3A had CKD-MBD (Figure 1).

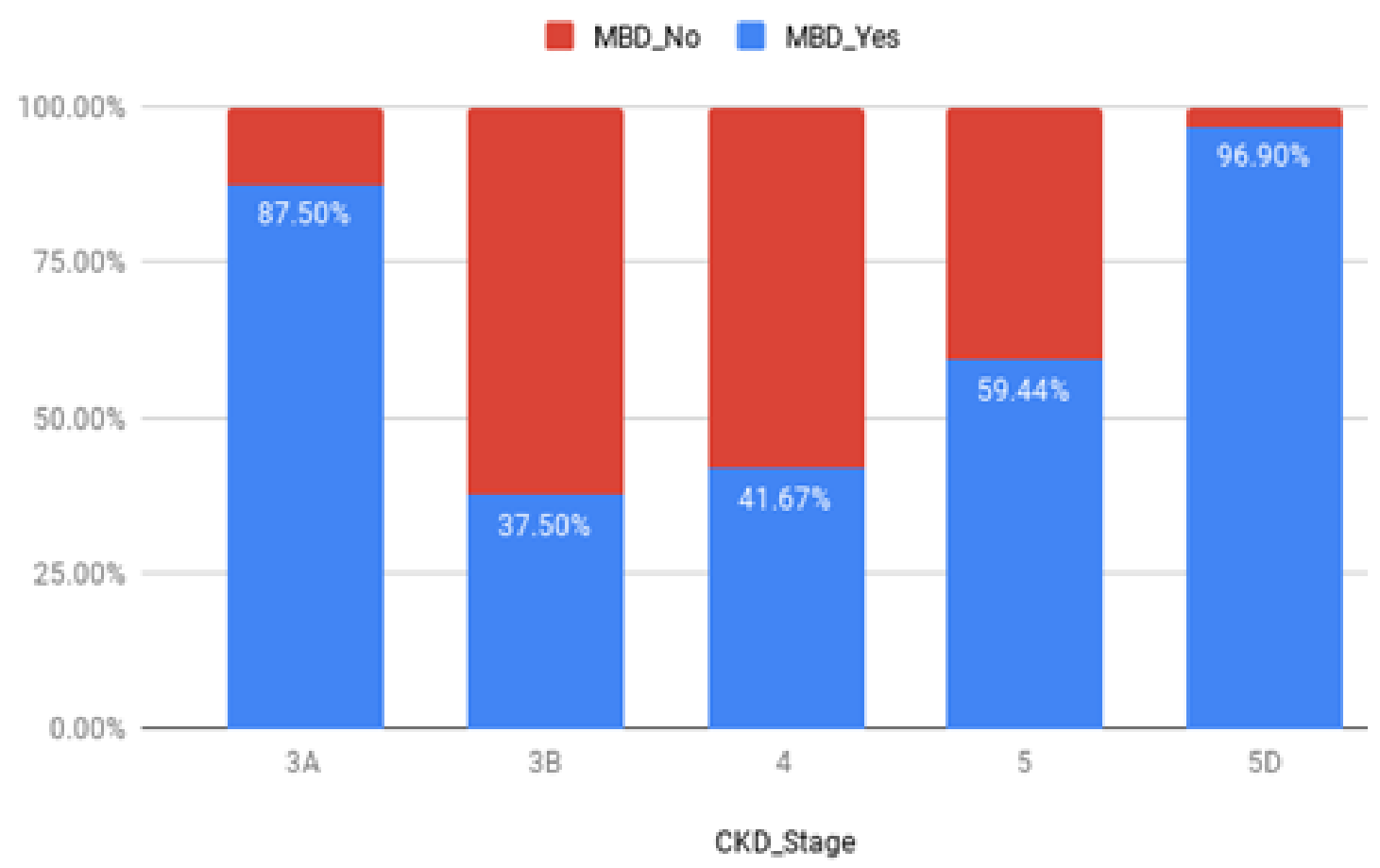

Figure 1: Distribution of participants by CKD-MBD 


\section{Types of MBD among patients with CKD-MBD}

CKD-MBD was defined based on an abnormality of calcium, phosphate or parathyroid hormone level. The most common MBD was hyperparathyroidism, found in $196(87.1 \%)$ of all patients with MBD, followed by hypocalcemia 174 (77.3\%) and hyperphosphatemia 82 (36.4\%), which was the least common (Figure 2).

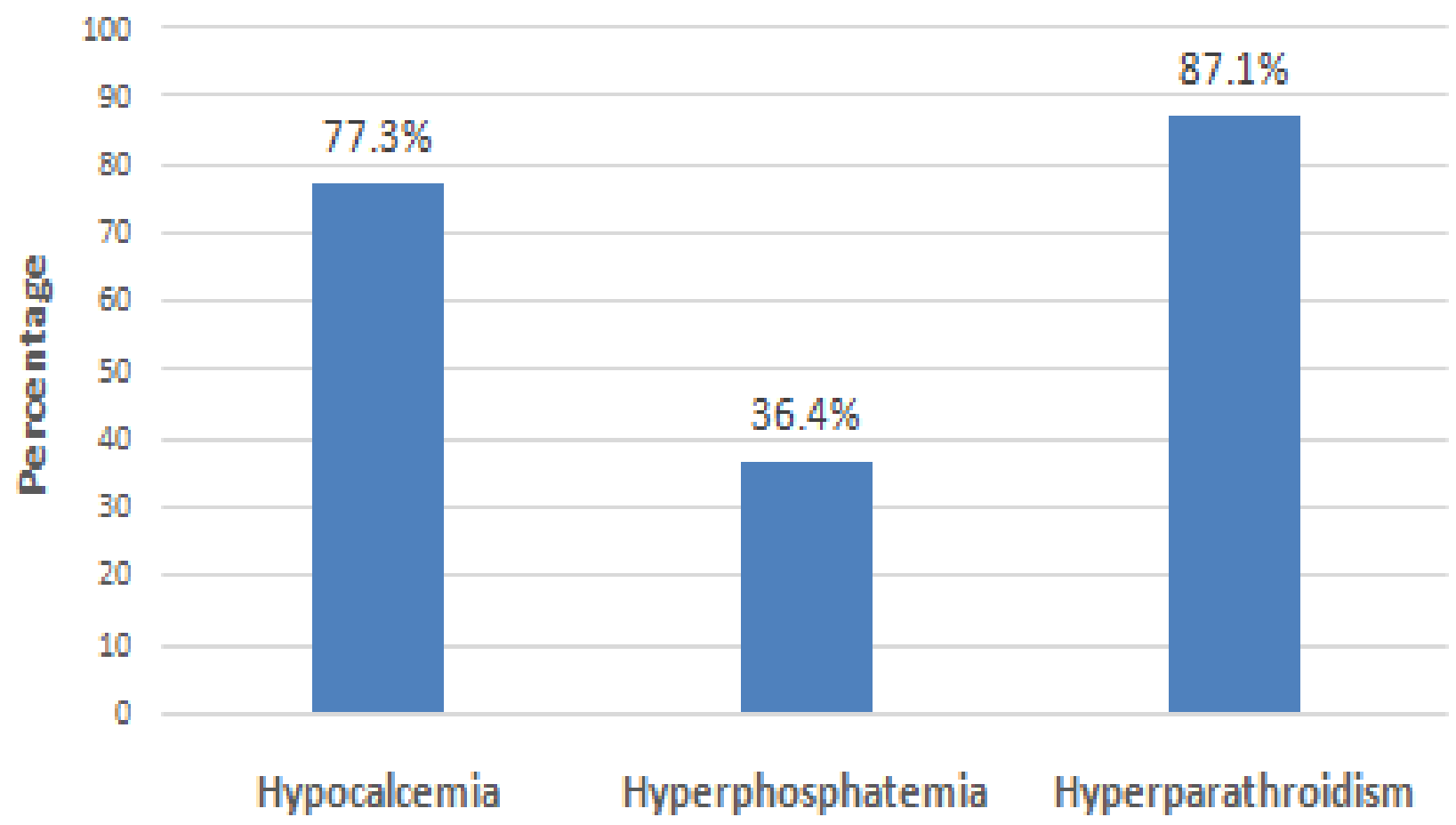

Figure 2: Types of MBD among patients with CKD-MBD 
Proportion of patients with CKD-MBD using calcium supplements, phosphate binders, calcium rich diet and low phosphate diet

Among the patients with CKD-MBD, 67 (29.8\%) were taking calcium supplements, 51 (22.7\%) were taking phosphate binders, $90(40.0 \%)$ were on a calcium rich diet and $75(33.7 \%)$ were on low phosphate diet (Figure 3).

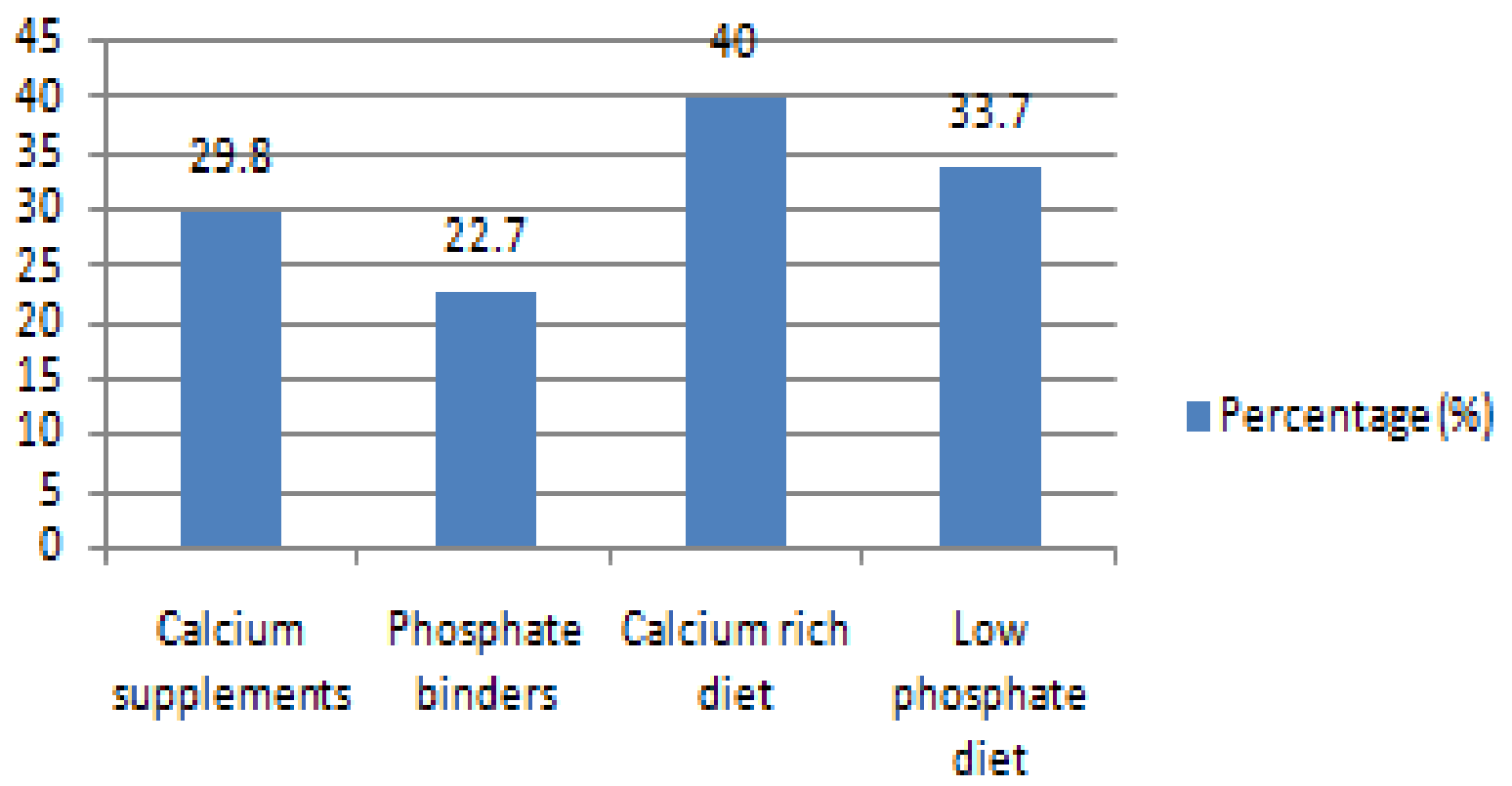

Figure 3: Proportion of patients with CKD-MBD using calcium supplements, phosphate binders, calcium rich diet and low phosphate diet 


\section{Factors associated with CKD-MBD}

In a univariate analysis, age >50years, use of calcium supplements and/or phosphate binders, being on dialysis, duration of CKD >2years and use of calcium rich diet and/or low phosphate diet were associated with CKD-MBD. Participants aged above fifty years had three fold high risk of developing CKD-MBD as compared to their younger counterparts [OR 3.32, (95\% C.I 1.616.82), $\mathrm{p}=0.001$ ]; participants who were on calcium supplements were two times more likely to have CKD-MBD [OR 2.56, (95\% C.I 1.50-4.37), $\mathrm{p}=0.001$ ]; participants who were on phosphate binders were three times more likely to have CKD-MBD [3.70, (95\% C.I 2.13-6.41), $\mathrm{p}=<0.001$ ]; participants who were on hemodialysis had more than sixty fold high risk of developing CKDMBD [OR 61.78, (95\% C.I 12.60-302.91), $\mathrm{p}=<0.001$ ] and participants who had a duration of CKD for more than two years had two fold high risk of developing CKD-MBD [OR 2.11, (95\% C.I 1.22-3.61), $\mathrm{p}=0.007]$.

In a multivariate analysis, all of these associations remained significant, although the odds ratios were smaller (Table 2). 
Table 2: Factors associated with CKD-MBD

\begin{tabular}{|c|c|c|c|c|}
\hline Variable & $\begin{array}{l}\text { Crude Odds } \\
\text { ratio (95\% } \\
\text { CI) }\end{array}$ & P-value & $\begin{array}{l}\text { Adjusted odds } \\
\text { ratios }(95 \% \mathrm{CI})\end{array}$ & P-value \\
\hline Age >50years & $\begin{array}{c}3.32(1.61- \\
6.82)\end{array}$ & 0.001 & $1.54(0.53-4.44)$ & 0.428 \\
\hline Male & $\begin{array}{c}1.125 \\
(0.651- \\
1.944)\end{array}$ & 0.673 & & \\
\hline Hypertension & $\begin{array}{c}1.352 \\
(0.371- \\
4.927)\end{array}$ & 0.647 & & \\
\hline Diabetes & $\begin{array}{c}0.806 \\
(0.435- \\
1.492)\end{array}$ & 0.493 & & \\
\hline $\mathrm{Ca}++$ supplements & $\begin{array}{c}2.56(1.50- \\
4.37)\end{array}$ & 0.001 & $1.29(1.11-1.51)$ & 0.001 \\
\hline Phosphate binders & $\begin{array}{c}3.70(2.13- \\
6.41)\end{array}$ & $<0.001$ & $\begin{array}{c}1.46(1.09- \\
21.00)\end{array}$ & 0.001 \\
\hline On dialysis & $\begin{array}{c}61.78(12.60 \\
-302.91)\end{array}$ & $<0.001$ & $\begin{array}{c}13.39(5.02- \\
35.71)\end{array}$ & $<0.001$ \\
\hline $\begin{array}{l}\text { CKD-Duration } \\
\text { (>2years) }\end{array}$ & $\begin{array}{c}2.11(1.22- \\
3.61)\end{array}$ & 0.007 & $1.18(0.37-3.73)$ & 0.776 \\
\hline $\mathrm{Ca}++$ rich diet & $\begin{array}{c}21.00(8.16- \\
54.07)\end{array}$ & $<0.001$ & $1.83(1.60-2.11)$ & 0.001 \\
\hline Low phosphate diet & $\begin{array}{c}148(20.18- \\
1085.45)\end{array}$ & $<0.001$ & $1.97(1.68-2.32)$ & 0.001 \\
\hline
\end{tabular}




\section{Discussion}

In this study, we found the prevalence of CKD-MBD to be $75 \%$ and the most common form of MBD was hyperparathyroidism $(87.10 \%)$, followed by hypocalcemia $(77.3 \%)$ and hyperphosphatemia (36.4\%). The prevalence of CKD-MBD found in our study was quite comparable with the prevalence found in patients with end-stage renal disease at Jordan University Hospital, which showed a prevalence of $70 \%$ [12]. However, it was higher than the estimated prevalence of CKD-MBD seen in studies done in Nigeria, Israel, Czech Republic and Kenya whereby they reported prevalence of 58\%, 33.3\%, 57\% and 22.4\% respectively [13-16]. The possible explanations of the high burden of CKD-MBD found in our study population could be the fact that a large proportion $(90.7 \%)$ of our study population was CKD stage 5 and only a small proportion of our study participants were on measures to prevent CKD-MBD such as the use of calcium supplements and/or phosphate binders and the minority were on calcium rich diet and/or low phosphate diet.

Several studies on the association between bone mineral disorder and renal outcomes have also found secondary hyperparathyroidism to be the most common form of metabolic bone disease among patients with CKD [1, 13-19]. This is because as the kidney function declines, there is a decrease in phosphate elimination, hyperphosphatemia and a hypocalcemia, all of which stimulate the secretion of parathyroid hormone in an attempt to normalize ionized calcium and serum phosphate levels [3].

Use of calcium supplements, phosphate binders, calcium rich diet and low phosphate diet were significantly associated with CKD-MBD mainly because patients with CKD-MBD had a declined kidney function, which lead them to have very low calcium levels and being unable to excrete phosphate from their body which necessitated them to be placed on calcium supplements and phosphate binders respectively. Although a study done by York Pei et al on the risk factors for renal osteodystrophy had found infrequent use of calcium and phosphate binders, were associated with metabolic bone disease, which is not the case in this study because being on these measures was a marker of having CKD-MBD [4].

Similar to our study, CKD-MBD has been reported to be a major complication in patients undergoing dialysis [1, 2, 12-20]. This can be explained by the fact that patients on dialysis have a progressive deterioration in mineral homeostasis which manifest as disruption serum 
concentration of phosphorus, calcium and parathyroid hormone level. These mineral biomarkers are important in the regulation of bone modeling and remodeling.

Our study has several limitations. First, being a single center study at a tertiary hospital, our study participants were mainly an advanced CKD population. Therefore, our study results might not be applicable in earlier stage CKD patients. Second, the lack of bone biopsy and bone scan for pathologic confirmation and determination of the risk of bone fracture are also limitations to this study. Third, we did not measure vitamin D and FGF-23 levels.

\section{Conclusion and Recommendations}

This study has revealed a high prevalence of metabolic bone disease among patients with CKD, therefore there is a need for physicians to pay special attention to the biomarkers of this disabling condition, as well as providing proper treatment to this condition.

We recommend serial monitoring of calcium, phosphate and parathyroid hormone levels, which are recognized primary indicators of CKD-MBD as well as early introduction of calcimimetic drugs, vitamin D analogues and phosphate binders to those patients with severe and persistent abnormalities in these bone mineral biomarkers to further reduce the contributions of CKD-MBD to the morbidity and mortality in patients with CKD.

\section{Declarations}

\section{Abbreviation}

CKD: Chronic Kidney Disease; CKD-MBD: Chronic Kidney Disease-Metabolic Bone Disease eGFR: Estimated-Glomerular Filtration Rate; KDIGO: Kidney Disease Improving Global Outcome; MBD: Metabolic Bone Disease; MDRD: Modification of Diet in Renal Disease; MNH: Muhimbili National Hospital; PTH: Parathyroid hormone; OR: Odds Ratio

\section{Consent for publication}

Not applicable

\section{Availability of data and materials}

The datasets analyzed during this study are available from the corresponding author on reasonable request

\section{Competing interests}

The authors declare that they have no competing interests.

\section{Funding}


By the Tanzania government through the ministry of health as part of masters of Internal Medicine scholarship program. The funder had no role in research design data collection and data analysis.

\section{Authors' contribution}

FFM contributed to the conception and design of the study, data acquisition and entry, analyzed and interpreted the data, drafted original manuscript and revised the manuscript. MA contributed to design of the study, data analysis and also revised the manuscript. PR contributed to conception and design of the study, data validation and analysis, and also critically revised the manuscript. FFF contributed to design of the study, review, analysis and also critically revised the manuscript. All authors read and approved the final manuscript.

\section{Acknowledgement}

We thank all the staff members of the Nephrology unit at Muhimbili National Hospital for their tireless support during this study. We also thank all the study participants who have been involved in this research. Special thanks to Prof. Ellen Weber for reviewing the work.

\section{Authors' information}

\section{Felix Faustin Mungulluh}

MD, MMed Resident in Internal medicine at Muhimbili University of health and Allied Sciences.

\section{Paschal Ruggajo}

$\mathrm{MD}, \mathrm{PhD}$, Nephrologist: Senior Lecturer and Head of department of Internal medicine at Muhimbili University of health and Allied Sciences.

\section{Francis Furia}

MD: Nephrologist; Senior Lecturer in the department of Pediatrics and Child health; Muhimbili University of health and Allied Sciences.

\section{References}

1. Valson AT, Sundaram M, David VG, Deborah MN, Varughese S, Basu G, Mohapatra A, Alexander S, Jose J, Roshan J, Simon B. Profile of incident chronic kidney disease related-mineral bone disorders in chronic kidney disease Stage 4 and 5: A hospital based cross-sectional survey. Indian journal of nephrology. 2014 Mar;24(2):97. 
2. Sanusi AA, Arogundade FA, Oladigbo M, Ogini LM, Akinsola A. Prevalence and pattern of renal bone disease in end stage renal disease patients in Ile-Ife, Nigeria. West African journal of medicine.2010;29(2).

3. Eknoyan G, Levin A, Levin NW. Bone metabolism and disease in chronic kidney disease. American Journal of Kidney Diseases. 2003 Oct 1;42:1-201.

4. Pei Y, Hercz G, Greenwood C, Serge G, Manuel A, Saiphoo C, Fenton S, Sherrard D. Risk factors for renal osteodystrophy: a multivariant analysis. Journal of Bone and Mineral Research. 1995 Jan;10(1):149-56.

5. Moe SM, Drueke TB. Management of secondary hyperparathyroidism: the importance and the challenge of controlling parathyroid hormone levels without elevating calcium, phosphorus and calcium-phosphorus product. American journal of nephrology. 2003;23(6):369-79.

6. Pei Y, Hercz G. Low turnover bone disease in dialysis patients. In Seminars in Dialysis 1996 Jul (Vol. 9, No. 4, pp. 327-331). Oxford, UK: Blackwell Publishing Ltd.

7. Malluche HH, Ritz E, Lange HP, Kutschera J, Hodgson M, Seiffert U, Schoeppe W. Bone histology in incipient and advanced renal failure. Kidney international. 1976 Apr $1 ; 9(4): 355-62$.

8. de Francisco AL. Secondary hyperparathyroidism: review of the disease and its management. Clinical therapeutics. 2004 Dec1;26(12):1976-93.

9. Kidney Disease: Improving Global Outcomes (KDIGO) CKD-MBD Work Group. KDIGO clinical practice guideline for the diagnosis, evaluation, prevention, and treatment of Chronic Kidney Disease-Mineral and Bone Disorder (CKD-MBD). Kidney international. Supplement. 2009 Aug(113):S1.

10. Payne RB, Little AJ, Williams RB, Milner JR. Interpretation of serum calcium in patients with abnormal serum proteins. Br Med J. 1973 Dec 15;4(5893):643-6.

11. Hunsicker LG, Adler S, Caggiula A, England BK, Greene T, Kusek JW, Rogers NL, Teschan PE, Beck G, Modification of Diet in Renal Disease Study Group. Predictors of the progression of renal disease in the Modification of Diet in Renal Disease Study. Kidney international. 1997 Jun 1;51(6):1908-19. 
12. Younes NA, Wahbeh A, Mahafzah W, Ailabouni W, Al-Mansour M, Hamzah Y, Sroujieh AS. The spectrum of bone disease in Jordanian hemodialysis patients. Saudi medical journal. 2006 May1;27(5):667.

13. Boner G, Olah AJ, Weiss A, Seelenfreud MZ, Rosenfeld JB. Renal osteodystrophy in end-stage renal failure patients in Israel.Israel journal of medical sciences. 1983 Jul;19(7):608-13.

14. Jaros A, Zadrazil J, Konecny K, Kosatikova Z, Zahalkova J, Vrublosky P, Krejci K. Single photon bone densitometry in hemodialysis patients. Biomedical papers. $1999 \mathrm{Jul}$ 1.

15. Abdu A, Abdu A, Arogundade FA. Prevalence and pattern of CKD-MBD among hemodialysis patients in Kano, Northwest Nigeria. Annals of African Medicine. 2019 Oct;18(4):191.

16. Mugera AN. Prevalence and patterns of hyperparathyroidism and mineral bone disease in patients with chronic kidney disease at Kenyatta National Hospital. University of Nairobi; 2013.

17. Ghosh B, Brojen T, Banerjee S, Singh N, Singh S, Sharma OP, Prakash J. The high prevalence of chronic kidney disease-mineral bone disorders: A hospital-based crosssectional study. Indian journal of nephrology. $2012 \mathrm{Jul} ; 22(4): 285$.

18. Jat JA, Mal P, Kumar D. Renal Osteodystrophy in End Stage Renal Failure Patients on Maintenance Haemodialysis. J ClinExpNephrol. 2016;1:25.

19. Seck SM, Dahaba M, Ka EF, Cisse MM, Gueye S, Tal AO. Mineral and bone disease in black african hemodialysis patients: a report from senegal. Nephro-urology monthly. 2012;4(4):613.

20. Buargub MA, Nabulsi MF, Shafeh TA. Prevalence and pattern of renal osteodystrophy in chronic hemodialysis patients: a cross sectional study of 103 patients. Saudi Journal of Kidney Diseases and Transplantation. 2006 Jul 1;17(3):401. 